\title{
Dynamical Properties of a Plastic Neural Network Model for Tinnitus Therapy and Inhibition of Oscillation Using Noise Stimulus
}

\author{
Ken’ichi Fujimoto*, Hirofumi Nagashino*, Yohsuke Kinouchi**, Ali A. Danesh ${ }^{\dagger}$, and Abhijit S. Pandya ${ }^{\ddagger}$
}

\begin{abstract}
Tinnitus is the perception of phantom sounds in the ears or in the head. Sound therapy techniques for tinnitus have been proposed. To account for mechanisms of tinnitus generation and the clinical effects of sound therapies from the viewpoint of neural engineering, we have proposed a plastic neural network model for the human auditory system. We found that this model has a bistable state, i.e., a stable oscillatory state and a stable equilibrium (non-oscillatory) state coexist at a certain parameter region. We also found that the oscillation can be inhibited by supplying sinusoidal stimulus, which is hypothesized as sound for treatment of tinnitus, to the model. By hypothesizing that the oscillation and the equilibrium correspond to generation and inhibition of tinnitus, respectively, we reported that these phenomena could explain the fact that the habituated human auditory system temporarily halts perception of tinnitus following sound therapy. This paper describes dynamical properties of the model and inhibition of the oscillation for two kinds of noise stimuli which correspond to sound for treatment of tinnitus in clinical. Through numerical simulations we found that adequate noise stimulus can inhibits the oscillation.
\end{abstract}

\section{INTRODUCTION}

Tinnitus is the perception of phantom sounds in the ears or in the head. A mechanism of tinnitus generation has been hypothesized from the viewpoint of general neurophysiology[1]. There are two typical sound therapy techniques where those who suffer from tinnitus hear these therapeutic sounds for several hours a day[2]: TM (Tinnitus Masking) technique and TRT (Tinnitus Retraining Therapy). Band noise and white noise are actually provided to the sufferers from tinnitus as therapeutic sound in TM and TRT, respectively. The sound therapies have the clinical effect that the sufferers temporarily stop perceiving tinnitus after the treatment.

The purpose of our study is to explain mechanisms of tinnitus generation and the clinical effect of the sound therapies from the viewpoint of neural engineering. Accrodingly we have proposed a plastic neural network model for the human auditory system[3], [4]. We already reported that an oscillatory state and an equilibrium (non-oscillatory) state

*K. Fujimoto and H. Nagashino are with School of Health Sciences, Faculty of Medicine, The University of Tokushima, Tokushima 770-8509 Japan \{fujimoto, nagasino\}@medsci.tokushima-u.ac.jp

**Y. Kinouchi is with Department of Life System, Institute of Technology and Science, The University of Tokushima, Tokushima 770-8506 Japan kinouchidee.tokushima-u.ac.jp

${ }^{\dagger}$ A. A. Danesh is with Department of Communication Sciences and Disorders, Florida Atlantic University, Boca Raton, FL 33431-0991 USA danesh@fau. edu

$¥$ A. S. Pandya is with College of Engineering and Computer Science, Florida Atlantic University, Boca Raton, FL 33431-0991 USA pandya@fau.edu coexist at a certain parameter region, and the oscillatory state is inhibited into the equilibrium by supplying sinusoidal stimulus[5]. By hypothesizing that the oscillation and the equilibrium correspond to generation and inhibition of tinnitus, respectively, we reported that these phenomena could explain the fact that the habituated human auditory system temporarily halts perception of tinnitus following sound therapy. However, we have not discussed a mechanism that the oscillation is inhibited by reducing the value of the plastic coupling. Moreover, the inhibition effect of noise stimuli, which correspond to actual therapeutic sounds, have not been investigated.

In this paper we investigate dynamical properties of the model to clarify the mechanism of inhibition of the oscillation. This paper also describes inhibition of the oscillation using two kinds of noise stimuli which correspond to actual therapeutic sounds for tinnitus.

\section{Plastic NeURAL NETWORK MODEL}

\section{A. Model description}

The human auditory system has a peripheral neural system and a central neural system as seen in Fig. 1(a). Hair cells at the cochlea is in the peripheral neural system and transforms acoustic vibrations received by the ear into neural signals. The neural signals reach the cerebral limbic system through the cochlear nucleus, the superior olivary complex, the inferior colliculus, the medical geniculate body, and the auditory cortex; and subsequently the brain perceives the neural signals as sound. When cochlear dysfunction occurs, abnormal neural signals from the cochlea causes abnormality in the central neural system. Consequently, tinnitus can be triggered. Note that the cochlear nucleus has an efferent neural bundle to control the sensitivity of the outer hair cells[6].

We have proposed a conceptual neural network model to account for tinnitus generation and its inhibition from the viewpoint of neural engineering[3], [4]. Figure 1(b) shows the proposed neural network model of the human auditory system. The cochlea is represented by an aggregate of excitatory neurons " $\mathrm{E}_{1}$ ". This has an incoming signal, $S$, which is associated with an acoustic vibration (sound signal). The central auditory pathway is simply represented as a neural oscillator which consists of an aggregate of excitatory neurons and an aggregate of inhibitory neurons: " $\mathrm{E}_{2}$ " and "I". The neural coupling from the $j$-th aggregate to the $i$-th aggregate is expressed by the positive constant $C_{i j}$, $(i, j \in\{1,2, I\})$. However, from the fact the efferent auditory pathway controls the sensitivity of outer hair cells[6], we 
hypothesize that the coupling from $E_{2}$ to $E_{1}$ is plastic, i.e., $C_{12}$ is one of variables. Accordingly the dynamics of the model is described by

$$
\begin{aligned}
\frac{d x_{1}}{d t} & =\left(-x_{1}+C_{12} Z_{2}+S\right) / \tau_{1} \\
\frac{d x_{2}}{d t} & =\left(-x_{2}+C_{21} Z_{1}-C_{2 I} Z_{i}\right) / \tau_{2} \\
\frac{d x_{I}}{d t} & =\left(-x_{I}+C_{I 2} Z_{2}\right) / \tau_{I} \\
\frac{d C_{12}}{d t} & =\left(-C_{12}+b Z_{1} Z_{2}+C_{0}\right) / \tau_{c}
\end{aligned}
$$

where $z_{j}$ is the output of the $j$-th aggregate, which is given by

$$
z_{j}=(2 / \pi) \tan ^{-1}\left(x_{j}\right)
$$

The $x_{j}$ and $\tau_{j}$ are the membrane potential and the time constant of the $j$-th aggregate, respectively. The $C_{0}, b$, and $\tau_{c}$ are also positive constants which denote the equilibrium of $C_{12}$ under $z_{1} z_{2}=0$, the efficiency of strengthening the synaptic coupling based on Hebb's hypothesis[7], and the time constant of $C_{12}$, respectively.

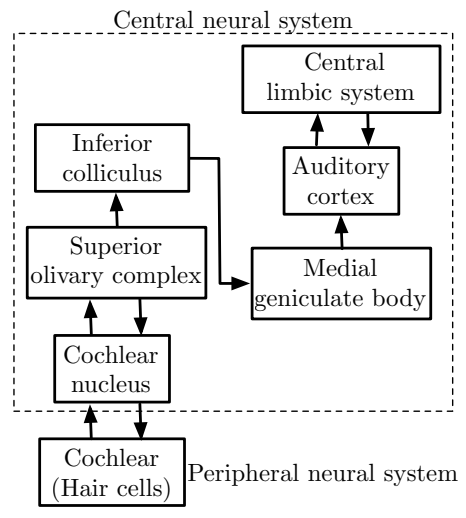

(a) The human auditory system

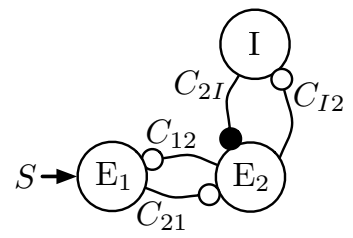

(b) A neural network model
Fig. 1. Schematic diagram of the human auditory system and its a conceptual neural network model[4].

\section{B. Oscillation and non-oscillation}

We fixed the parameters in Eqs.(1)-(4) such that $\tau_{1}=0.01$, $\tau_{2}=0.01, \quad \tau_{I}=0.02, \quad \tau_{c}=0.5, \quad C_{21}=10, \quad C_{2 I}=10, \quad C_{I 2}=20$, $C_{0}=3.0$, and $b=20$. We can easily find an equilibrium point in the dynamical system: $\left(x_{1}, x_{2}, x_{I}, C_{12}\right)=(0,0,0,3)$. Figure 2(a) shows the behavior of a solution which starts at the initial point $\left(x_{1}, x_{2}, x_{I}, C_{12}\right)=(5,-5,5,7)$. This point is at the vicinity of the equilibrium point. The behavior eventually converges to the equilibrium point, i.e., the stable state according to the Lyapunov stability theory. On the other hand, we illustrate the behavior of another solution in Fig. 2(b); its initial point is $\left(x_{1}, x_{2}, x_{I}, C_{12}\right)=(-5,-1$, $-6,9)$, which is far away from the equilibrium point. As a result, the behavior converges to the oscillatory state, and its fundamental frequency is about $15 \mathrm{~Hz}$. Through other simulations we observed that the behavior converges to the oscillatory state whenever the initial values are arranged at any point at the vicinity. Therefore, the oscillatory state is stable, and the model has the bistable state under the same parameters. We hypothesize that the oscillation and the nonoscillation correspond to the state of perceiving tinnitus and disappearance of tinnitus, respectively.

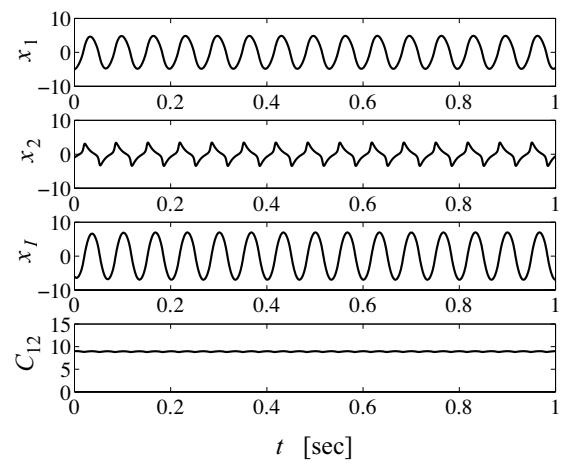

(a) Non-oscillatory state

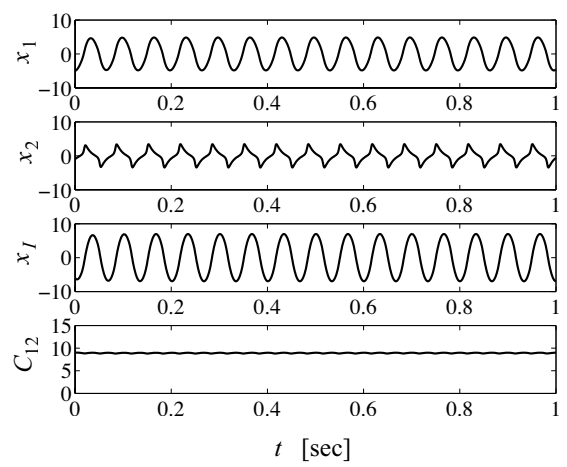

(b) Oscillatory state

Fig. 2. Non-oscillatory state and oscillatory state observed under the same parameters[4].

\section{NUMERICAL RESULTS}

\section{A. Attractive region of non-oscillation}

We illustrate attractive regions of the non-oscillation (equilibrium point) on the $\left(x_{1}, x_{2}\right)$-plane for respective parameters of $C_{12}$ in Fig. 3. In the figures, a solution in Eqs.(1)-(4) necessarily converges to the equilibrium point, $\left(x_{1}, x_{2}, x_{I}\right.$, $\left.C_{12}\right)=(0,0,0,3)$, when its initial point is in the black region. Notice that these results show dynamical properties of the non-plastic neural network model which is described by Eqs.(1)-(3), i.e., $C_{12}$ is constant at 8.0 or 6.7. As the results, the model has the dynamical property that attractive region of the non-oscillation is expanded by reducing the value of $C_{12}$. In addition, any oscillatory behavior in such region eventually settles down in non-oscillatory state without external stimulus according to the dynamical property of the model. Therefore, to inhibit the oscillation it is important that the states of $\left(x_{1}, x_{2}, x_{I}, C_{12}\right)$ change into such an attractive region of non-oscillation by external stimulus.

\section{B. Inhibition of oscillation by external noise stimulus}

We already reported that the oscillation can be inhibited by supplying adequate sinusoidal stimulus[5]. However, in- 


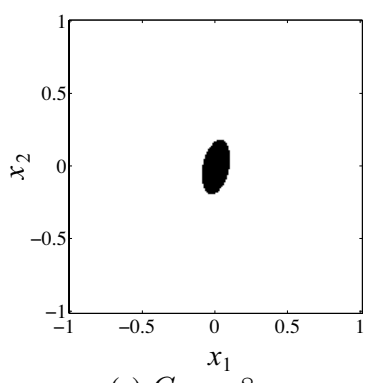

(a) $C_{12}=8$

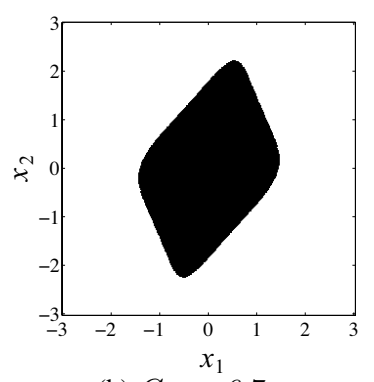

(b) $C_{12}=6.7$
Fig. 3. Attractive regions of the non-oscillation (equilibrium point) at all constant couplings, i.e., $C_{12}$ is constant. The initial value of $x_{I}$ is fixed to zero.

hibition effects of noise stimuli have not been investigated. In this paper, let us demonstrate inhibition of the oscillation by two kinds of stimuli: band noise and white noise which are actually used as therapeutic sound in clinical.

1) Band noise stimulus: Band noise stimulus is used in $\mathrm{TM}$ approach[2]. In this approach the desired noise is a band of noise with a frequency emphasis that approximates the frequency of perceived tinnitus. The frequency (pitch match) of tinnitus can range from low frequencies to high frequencies in different individuals. Most of the tinnitus sufferers perceive tinnitus at high frequencies between $2-8 \mathrm{kHz}$. In this experiment, we hypothesized that the fundamental frequencies of perceived tinnitus are $2,4,6$, and $8 \mathrm{kHz}$; we subsequently employed a band noise which is generated from Gaussian white noise through a band pass filter which operates between each fundamental frequency with $\pm 5 \%$ margin. We also adjusted its RMS (Root Mean Square) to about 400 , and the band noise was applied for the duration of $2 \leq t \leq 8$. Figures $4-7$ show successful results for inhibition of the oscillation. The left column figures show the waveforms of $x_{1}, x_{2}, x_{I}, C_{12}$, and $S$, respectively; their power spectra are illustrated at the right column figures, respectively. From the results, the value of $C_{12}$ gradually reduces from the point of applying stimulus, and consequently the oscillation is inhibited. On the other hand, as seen in Fig. 8 the value of $C_{12}$ is almost unchangeable for band noise with low power $(\mathrm{RMS}=10)$, and consequently the oscillation is kept through whole simulation time. Therefore, inhibition of the oscillation by band noise stimulus with appropriate RMS value is possible.

2) Gaussian white noise stimulus: White noise stimulus is used in TRT[2]. Let us add Gaussian white noise for the duration of $2 \leq t \leq 8$. We obtained two simulation results for different RMS values in Figs. 9-10. Figure 9 shows a result at $\mathrm{RMS}=10$ that the oscillatory states are inhibited; is was also observed that the value of $C_{12}$ gradually decreases after supplying the stimulus. On the other hand, Fig. 10 shows an unsuccessful result for large power stimulus $(\mathrm{RMS}=100)$. In this simulation, the value of $C_{12}$ increases for the duration of applying the stimulus. Hence, as in the case of band noise stimulus, to inhibit the oscillation we have to adjust the RMS of stimulus to appropriate value.

\section{Conclusions}

In this paper we investigated attractive regions of nonoscillation for different values of the plastic coupling coefficient. It was clarified that its region is expanded by depending on decrease of the value of the plastic coupling coefficient. Subsequently we demonstrated inhibition of the oscillation using two kinds of noise stimuli: band noise and Gaussian white noise which are used as therapeutic sounds for tinnitus in clinical. Through several numerical simulations we found that either noise stimulus with appropriate RMS value can inhibit the oscillation. Therefore, by hypothesizing that the decrement of the plastic coupling value corresponds to the habituation of the human auditory system for tinnitus, these phenomena could account for the fact that the habituated human auditory system temporarily halts perception of tinnitus following sound therapy using noise stimulus.

\section{REFERENCES}

[1] P. J. Jastreboff, Phantom auditory perception (tinnitus): mechanisms of generation and perception, Neuroscience Research, vol. 8, no. 4, 1990, pp. 221-254.

[2] J. A. Henry, M. A. Schechter, T. L. Zaugg, S. Griest, P. J. Jastreboff, J. A. Vernont, C. Kaelin, M. B. Meikle, K. S. Lyons, and B. J. Stewart, Outcomes of clinical trial: tinnitus masking versus tinnitus retraining therapy, J. of the American Academy of Audiology, vol. 17, no. 2, 2006, pp. 104-132.

[3] K. Fujimoto, H. Nagashino, Y. Kinouchi, A. A. Danesh, and A. S. Pandya, "Analysis of a Neural Oscillator Model With Plasticity for Treatment of Tinnitus", Proc. of World Congress on Medical Physics and Biomedical Engineering, vol. 14, 2006, pp. 3413-3416.

[4] K. Fujimoto, H. Nagashino, Y. Kinouchi, A. A. Danesh, and A. S. Pandya, A Plastic Neural Network Model for Sound Therapy of Tinnitus, IEEJ Trans. on Electrical and Electronic Engineering, (in press).

[5] K. Fujimoto, H. Nagashino, Y. Kinouchi, A. A. Danesh, and A. S. Pandya, "Oscillation and Its Inhibition in a Neural Oscillator Model for Tinnitus", Proc. of the 28th IEEE-EMBS Annual International Conference, 2006, pp. 5547-5550.

[6] J. H. Martin, Neuroanatomy Text and Atlas Third Ed., McGraw-Hill Medical, 2003.

[7] D. O. Hebb, The organization of behavior: A neuropsychological theory, New York: John Wiley \& Sons, 1949.
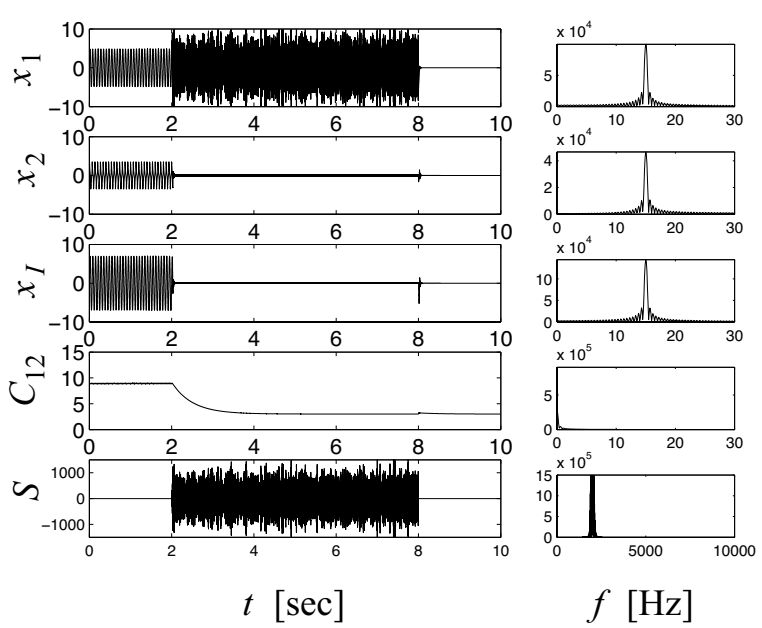

Fig. 4. Inhibition of oscillation by band noise stimulus with the band between $2 \mathrm{kHz} \pm 5 \%$ margin and 400 RMS. 

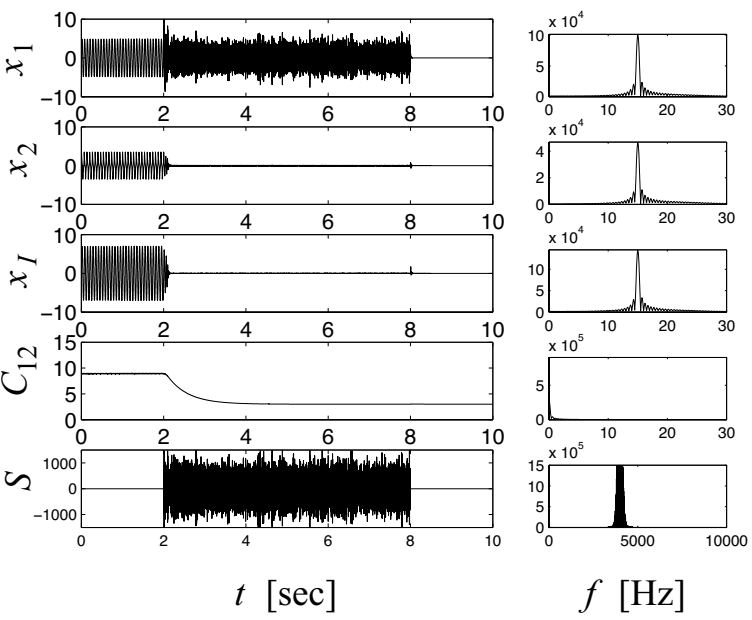

Fig. 5. Inhibition of oscillation by band noise stimulus with the band between $4 \mathrm{kHz} \pm 5 \%$ margin and 400 RMS.
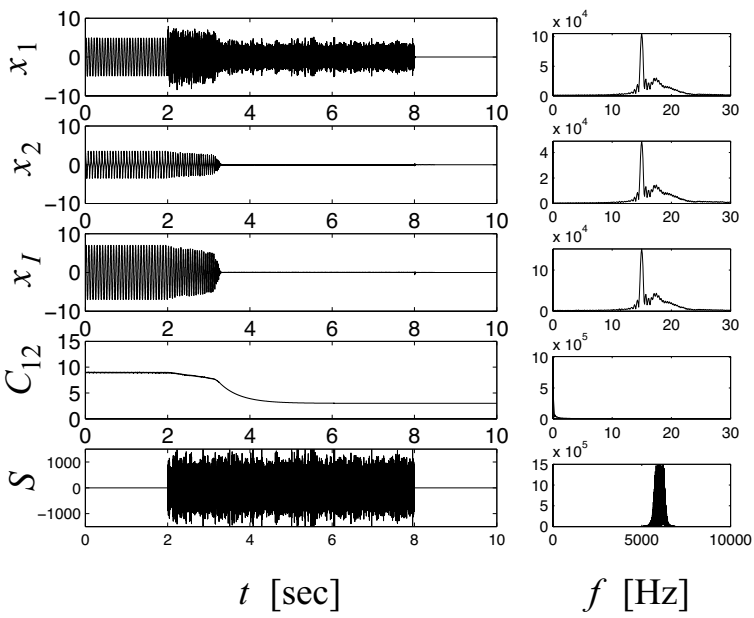

Fig. 6. Inhibition of oscillation by band noise stimulus with the band between $6 \mathrm{kHz} \pm 5 \%$ margin and $400 \mathrm{RMS}$.

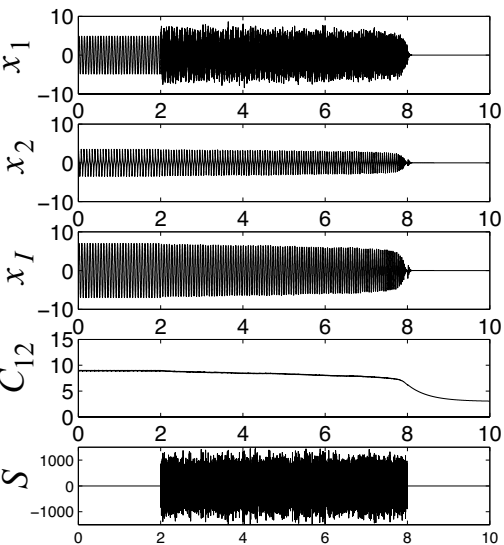

$t[\mathrm{sec}]$
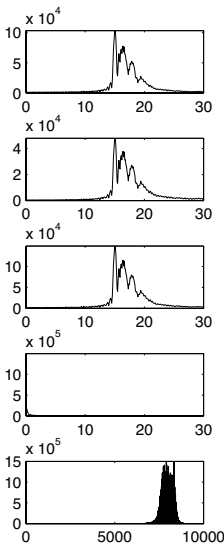

$f[\mathrm{~Hz}]$
Fig. 7. Inhibition of oscillation by band noise stimulus with the band between $8 \mathrm{kHz} \pm 5 \%$ margin and $400 \mathrm{RMS}$.

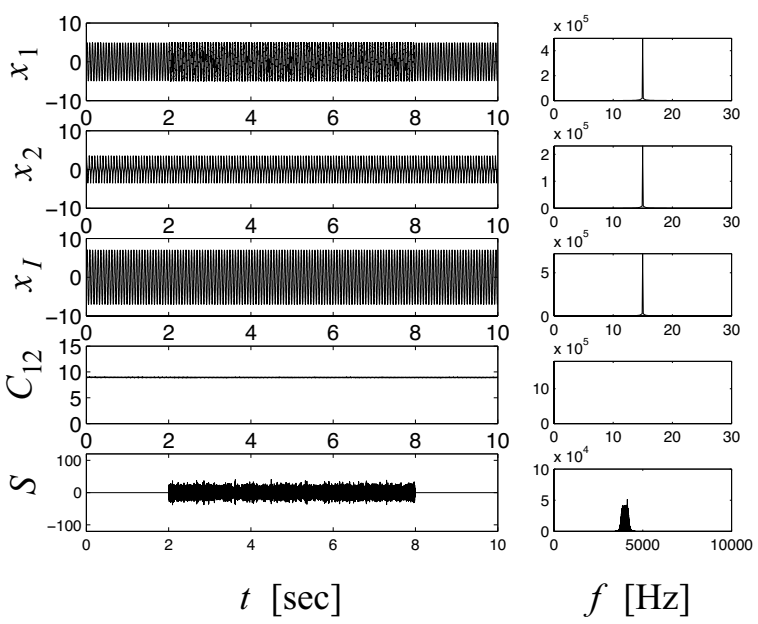

Fig. 8. An unsuccessful result for inhibition of oscillation by band noise stimulus with the band between $4 \mathrm{kHz} \pm 5 \%$ margin and $10 \mathrm{RMS}$.

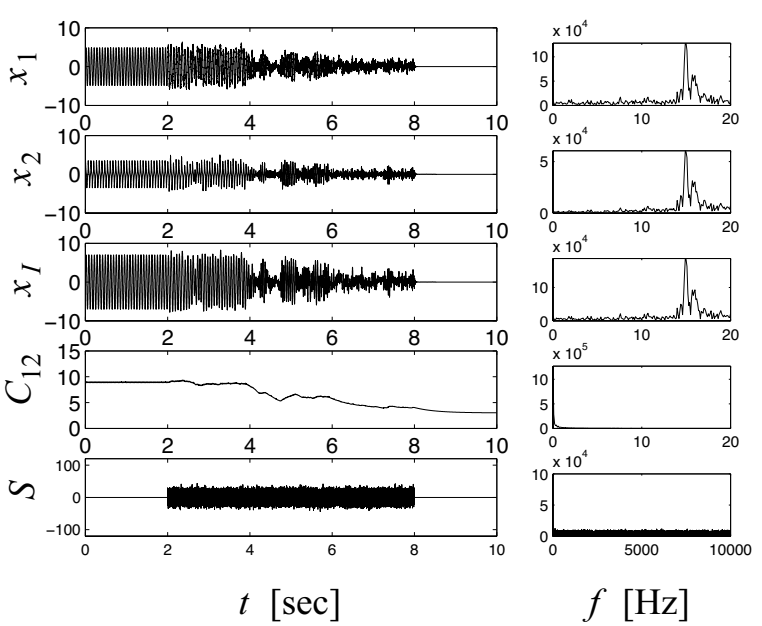

Fig. 9. A successful result for inhibition of oscillation by Gaussian white noise stimulus with 10 RMS.
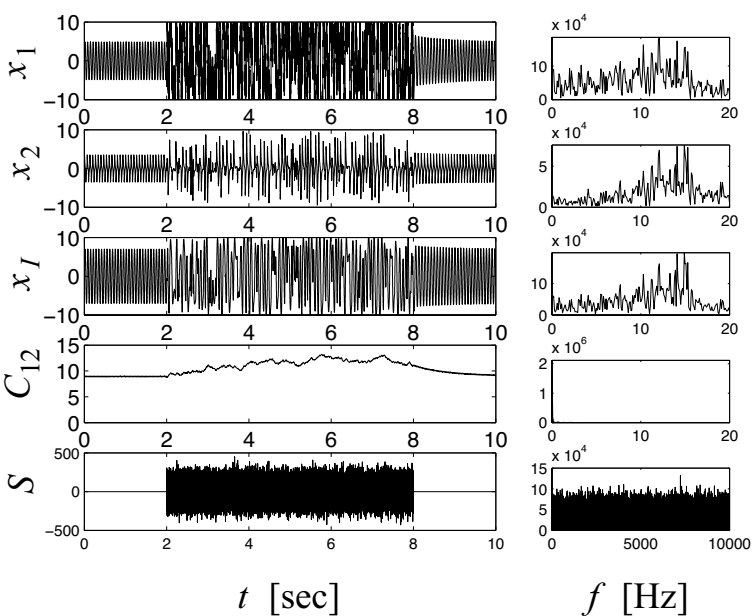

Fig. 10. An unsuccessful result for inhibition of oscillation by Gaussian white noise stimulus with 100 RMS. 